\title{
Hybrid Algorithm for Solving the Quadratic Assignment Problem
}

\author{
Mohammed Essaid Riffi, Fatima Sayoti* \\ LAROSERI Laboratory, Dept. of Computer Science, Faculty of Sciences, University of Chouaib \\ Doukkali, El Jadida (Morocco)
}

Received 1 June 2017 | Accepted 27 September 2017 | Published 20 October 2017

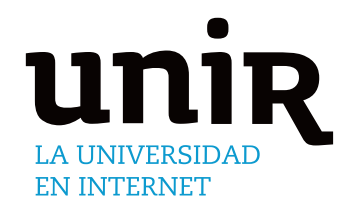

\section{ABSTRACT}

The Quadratic Assignment Problem (QAP) is a combinatorial optimization problem; it belongs to the class of NP-hard problems. This problem is applied in various fields such as hospital layout, scheduling parallel production lines and analyzing chemical reactions for organic compounds. In this paper we propose an application of Golden Ball algorithm mixed with Simulated Annealing (GBSA) to solve QAP. This algorithm is based on different concepts of football. The simulated annealing search can be blocked in a local optimum due to the unacceptable movements; our proposed strategy guides the simulated annealing search to escape from the local optima and to explore in an efficient way the search space. To validate the proposed approach, numerous simulations were conducted on 64 instances of QAPLIB to compare GBSA with existing algorithms in the literature of QAP. The obtained numerical results show that the GBSA produces optimal solutions in reasonable time; it has the better computational time. This work demonstrates that our proposed adaptation is effective in solving the quadratic assignment problem.

\section{KEYWORDS}

Combinatorial Optimization, Golden Ball Algorithm, Simulated Annealing, Quadratic Assignment Problem.

I: $10.9781 /$ ijimai.2017.10.003

\section{INTRODUCTION}

T, HE quadratic assignment problem (QAP) is one of the known classical combinatorial optimization problems, in 1976 Sahni and Gonzalez [1] proved that the QAP belongs to the class of NP-hard problems [1]. It was introduced for the first time by Koopmans and Beckmann in 1957 [2]; its purpose is to assign $n$ facilities to $n$ fixed locations with a given flow matrix of facilities and distance matrix of locations in order to minimize the total assignment cost. This problem is applied in various fields such as hospital layout [3], scheduling parallel production lines [4] and analyzing chemical reactions for organic compounds [5].

Many recent hybrid approaches have improved performance in solving QAP such as genetic algorithm hybridized with tabu search method [6], ant colony optimization mixed with local search method [7] and ant colony optimization combined with genetic algorithm and local search method [8]. Recently the hybrid algorithms are much proposed and used by many researchers to find optimal or near optimal solutions for the QAP.

In this paper we propose a new competitive approach when compared with other existing methods in the literature. The golden ball algorithm mixed with simulated annealing (GBSA) is considered here as a hybrid metaheuristic to apply in the quadratic assignment problem.

This work presents an efficient adaptation of GBSA algorithm to the quadratic assignment problem (QAP). This algorithm is based on the concept of soccer; it guides the search by simulated annealing [9] to

* Corresponding author.

E-mail address: fatimasayoti@gmail.com escape from the local optima. The suggested technique has never been proposed or tested with QAP. In this research we use some small, medium and large test problems for comparing our approach to other recent methods from literature. Our approach is able to explore effectively the search space; it reaches the known optimal solutions in less time.

The rest of this paper is structured as follows: In section I, Introduction. In section II, Quadratic assignment problem formulation. In section III, Methods. In section IV, Results and discussion. In section V, Conclusion.

\section{Quadratic Assignment Problem}

The QAP [1] can be defined as a problem of assigning $n$ facilities to $\mathrm{n}$ locations, with given flows between the facilities and given distances between the locations (Fig.1).

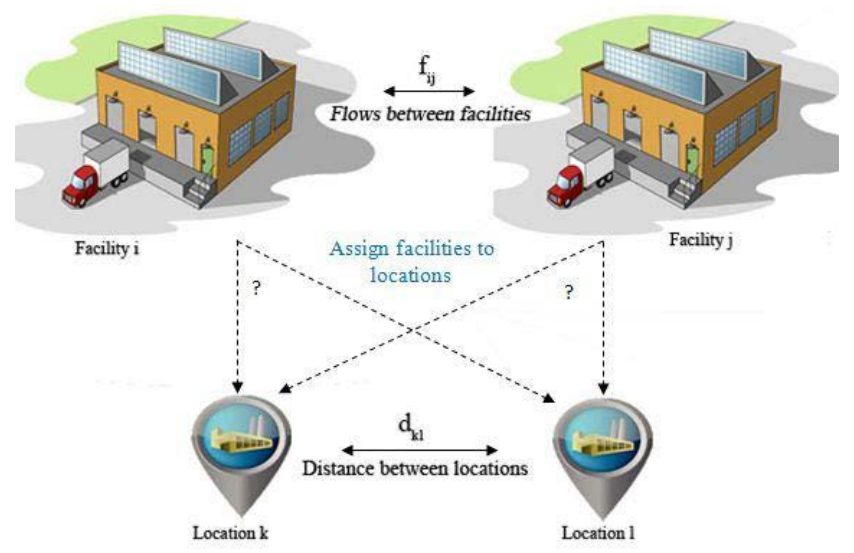

Fig. 1. Quadratic Assignment Problem. 
The purpose is to assign the facilities to the locations in such a way that the total cost is minimized. Each facility must be placed just at one location.

We consider two $\mathrm{n} \times \mathrm{n}$ matrices, the flow matrix $\mathrm{F}=\mathrm{f}_{\mathrm{ij}}$ and the distance matrix $\mathrm{D}=\mathrm{d}_{\mathrm{kl}}$. The QAP formulation is given as follows (1):

$$
\min _{\pi \in S_{n}} \operatorname{Cost}=\sum_{\mathrm{i}=1}^{\mathrm{i}=\mathrm{n}} \sum_{\mathrm{j}=1}^{\mathrm{j}=\mathrm{n}} \mathrm{f}_{\mathrm{ij}} \mathrm{d}_{\pi(\mathrm{i}) \pi(\mathrm{j})}
$$

$S_{n}$ is the set of all permutation of $\mathrm{n}$ elements $\{1,2, \ldots, \mathrm{n}\}$.

$\pi(\mathrm{i})$ and $\pi(\mathrm{j})$ are respectively locations of facilities $i$ and $j$, we suppose that $\pi(i)=k$ and $\pi(j)=l$.

$f_{i j} d_{\pi(i) \pi(j)}$ is the cost of assigning facility $i$ in location $k$ and facility $j$ in location $l$.

The objective function (Cost) must be minimized.

Several algorithms are usually used to solve the quadratic assignment problem:

- Exact algorithms such as branch and bound algorithm [10] and branch and cut algorithm [11].

- Metaheuristics such as genetic algorithm [12],[13],[14], tabu search method [15],[16],[17], simulated annealing algorithm [9], ant colony optimization [18] and particle swarm optimization [19].

In recent year, metaheuristic algorithms are used in solving the QAP more than the exact algorithms which are unable to solve the hard instances of QAP in a reasonable time. Many researchers compared between different metaheuristic algorithms for solving the QAP [20], [21].

\section{METHODS}

\section{A. Golden Ball Metaheuristic}

The GB technique is a metaheuristic proposed by E.Osaba et al. [22],[23]. It uses different principles of soccer to solve combinatorial optimization problems. The quality of this technique is demonstrated applying it to four combinatorial problems [23]: Asymmetric traveling salesman problem (ATSP) [24], Vehicle Routing Problem with Backhauls (VRPB) [25],[26], n-Queen Problem (NQP) [27], One-Dimensional Bin Packing Problem (BPP) [28].This algorithm is a promising metaheuristic to solve combinatorial optimization problems [23].

In Golden Ball algorithm, groups of solutions are considered as soccer teams which are composed of a fixed number of players, the captain of team plays the rule of the best solution of the group. Each team has a coach who determines the type of training to improve the efficiency of its team. There are two types of training: conventional training and custom training. As shown in Fig. 2, the concept of this method is based on four main phases: initialization phase, training phase, competition phase and transfer phase.

In the initialization phase, we set the value of the number of teams (NT) and the number of players per team (PT). We assign randomly to each team a coach.

In the training phase, all teams must train by following a specific type of training. The conventional training is the daily training of a team. When a team becomes unable to improve its capacities, in this case, it must follow a custom training.

In the competition phase, each team must compete with other team chosen randomly. The winning team receives three points, in the case of equality; both teams receive one point. The accumulated points will be used to order the teams in descending order.

In the transfer phase, we detect three cases of transfer:

Season transfer: during the season, all teams must be sorted in the descending order according to the strength value.

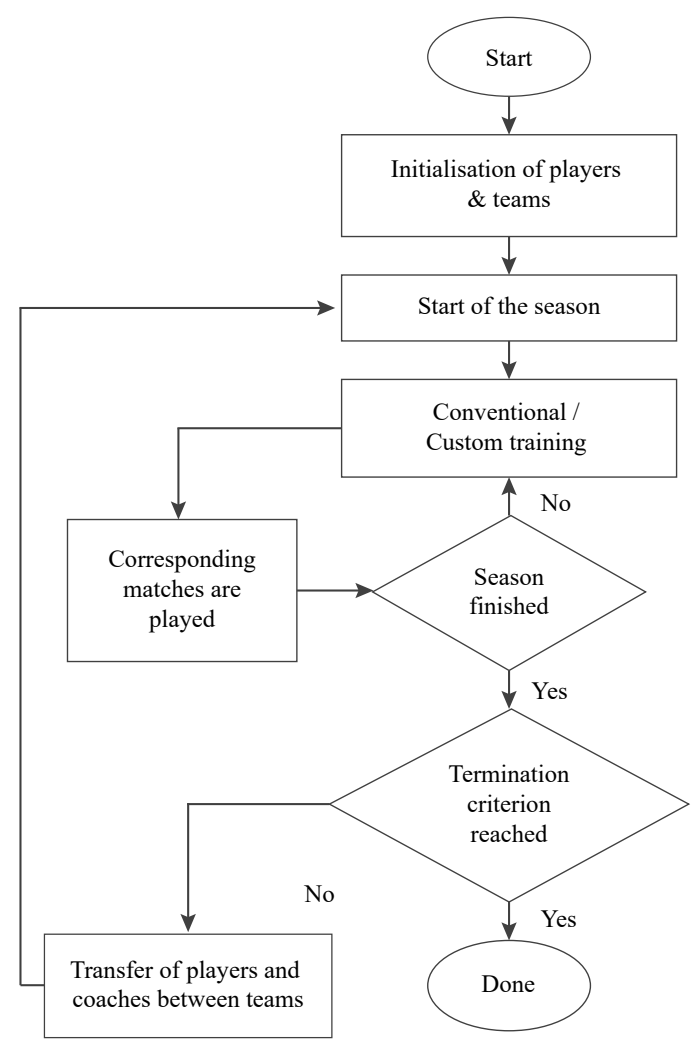

Fig. 2. Flowchart of Golden Ball algorithm.

The strength value is calculated using the following formula (2):

$\frac{\sum_{i=1}^{P T} q i j}{P T}$

$q_{i j}$ is the quality of player $i$ of team $j$

All teams exchange their players in this way: the best player of the first team must be replaced by the worst player of the last team. This worst player will be replaced by this best player.

The best player of the second team must be replaced by the worst player of the penultimate team. This worst player will be replaced by this best player and so forth.

Special transfer: When a player of a given team is unable to improve after a custom training, the team must exchange it with a player of another team chosen randomly.

Cessation of coaches: after having ordered all the teams in descending order according to their accumulated point, the weaker teams must change their conventional training by another randomly selected.

The GB algorithm was tested by E.Osaba et al. with four different combinatorial optimization problems [23]. The same technique was applied on the flow shop scheduling problem [29] and the job shop scheduling problem [30].

\section{B. Simulated Annealing Method}

The simulated annealing algorithm [14] is inspired by the physical annealing process which attempt to improve the quality of the solid by using at the beginning a high temperature $\mathrm{T}_{0}$ at which the solid is in a liquid state. With the slow decrease of the temperature $\mathrm{T}$ (cooling phase) the solid regains its solid form (Fig. 3). Metropolis et al. show how to generate a sequence of successive states of the solid. The new state is accepted if the energy produced by this change of state decreases; otherwise, it is accepted with a probability defined by the following equation (3). 
$p=e^{-\Delta E /\left(c_{b} \times T\right)}$

$c_{b}$ is Boltzmann constant

$\Delta E$ is the energy difference produced by this change of state

$T$ is the temperature of the solid

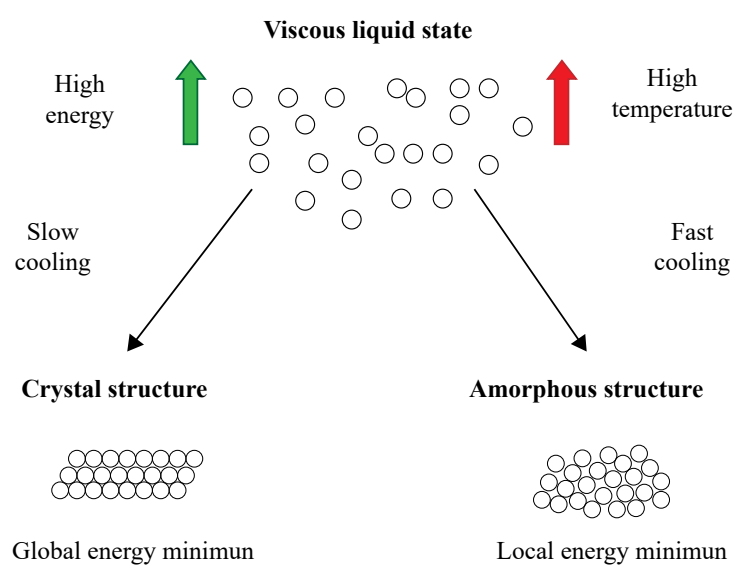

Fig. 3. Evolution of thermodynamic system.

The simulated annealing method [31] is one of the oldest algorithms; it is an iterative metaheuristic very used to solve combinatorial optimization problems in the continuous and discrete case. The strong point of this technique is to escape from the local minima and avoid the cyclic behavior. The performance of simulated annealing algorithm depends on a set of parameters which must be controlled. It means that the correct setting of the parameters produces satisfactory results.

\section{AdAPTATION OF GBSA Algorithm}

In the initialization phase we generate randomly the initial population of $\mathrm{NT} \times \mathrm{PT}$ solutions.

Each solution is represented in the following manner (Fig. 4):

\begin{tabular}{|c|c|c|c|c|c|c|c|c|c|c|}
\hline Locations & $\rightarrow$ & 2 & 7 & 4 & 5 & 6 & 3 & 9 & 8 & 1 \\
\hline
\end{tabular}

Fig. 4. Assign 9 facilities to 9 locations.

In the training phase, we used the following methods as conventional training functions:

2-opt [32], [33]: this iterative method is a local search algorithm, it repeatedly tries to improve the current assignment by exchanging two facilities.

Insertion method [34]: this method inserts a facility chosen randomly between two facilities.

Swapping mechanism [35]: this method swaps two parts selected randomly; the following figure (Fig. 5) explains the concept of this technique.

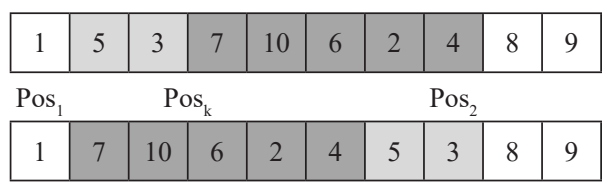

Fig. 5. Illustration of swapping mechanism.

As a custom training function the proposed adaptation used simulated annealing method [14],[31], it is used when the current solution is blocked in the local minima; it helps to accept some movement and escape from the local optimum.

\begin{tabular}{|c|}
\hline Simulated annealing steps \\
\hline S1:=Current solution \\
\hline $\begin{array}{l}\text { Generate a new solution based on the current solution. We used } \\
\text { the swap of two random locations }\end{array}$ \\
\hline $\mathrm{S} 2:=$ New solution \\
\hline $\mathrm{f}(\mathrm{S} 1):=$ cost of $\mathrm{S} 1$ \\
\hline$f(S 2):=$ cost of $S 2$ \\
\hline if $(\mathrm{f}(\mathrm{S} 2)<\mathrm{f}(\mathrm{S} 1))$ \\
\hline $\mathrm{S} 1:=\mathrm{S} 2$ \\
\hline Else \\
\hline Generate random number $\mathrm{r}$ in $[0,1)$ \\
\hline Calculate the value of $p$ \\
\hline$p=e^{-(f(S 2)-f(S 1)) /(T)}$ \\
\hline If $(r<p)$ \\
\hline $\mathrm{S} 1:=\mathrm{S} 2$ \\
\hline Decrease the temperature value \\
\hline Repeat all steps until $\mathrm{T}=0$. \\
\hline
\end{tabular}

\section{Results and Discussion}

The program was run 10 times on different instances of QAPLIB [36]. The GBSA algorithm was implemented in C language and compiled using Microsoft Visual Studio 2008, the program code was executed in computer with Genuine Intel( R ) $575 @ 2.00 \mathrm{GHz} 2.00$ GHz RAM 2,00 Go.

The program uses three parameters: NT (number of groups), PT (number of schedules per group) and $\mathrm{T}$ (temperature).

The parameters values in the table below (Table I) produce better results during the algorithm run.

$4 \times 5$ random solutions are sufficient to obtain good results.

TABLE I. PARAmeters VAlues

\begin{tabular}{cc}
\hline NT & 4 \\
PT & 5 \\
T & 40 \\
\hline \hline
\end{tabular}

At the high temperature, the simulated annealing method becomes unnecessary because proximally $50 \%$ of iterations accept decision at the high temperature [37]. In this paper we fixed the high temperature at 40 which is considered a symptom of fever in humans.

Table II represents the following information:

Optimal: Best known Solution

Best: Best permutation

NBest: The number of runs in which the algorithm reaches the best permutation

Worst: The worst permutation

Average: The average cost (= the sum of solutions cost obtained divided by 10)

The relative percentage deviation from the best known solution is calculated as follows (4):

$$
\mathrm{RPD}=\frac{\text { Average }- \text { Optimal }}{\text { Optimal }} \times 100 \%
$$

Time: Best time per seconds 
TABLE II. Numerical Results of the GBSA Algorithm

\begin{tabular}{|c|c|c|c|c|c|c|c|}
\hline Instance & Optimal & Best & Nbest & Worst & Average & $\%$ RPD & Time \\
\hline Bur26a & 5426670 & 5426670 & 10 & 5426670 & 5426670,00 & 0,00 & 0 \\
\hline Bur26b & 3817852 & 3817852 & 10 & 3817852 & 3817852,00 & 0,00 & 0 \\
\hline Bur26c & 5426795 & 5426795 & 10 & 5426795 & 5426795,00 & 0,00 & 1 \\
\hline Bur26d & 3821225 & 3821225 & 10 & 3821225 & 3821225,00 & 0,00 & 2 \\
\hline Bur26e & 5386879 & 5386879 & 10 & 5386879 & 5386879,00 & 0,00 & 1 \\
\hline Bur26f & 3782044 & 3782044 & 10 & 3782044 & 3782044,00 & 0,00 & 1 \\
\hline Bur26g & 10117172 & 10117172 & 10 & 10117172 & 10117172,00 & 0,00 & 0 \\
\hline Bur26h & 7098658 & 7098658 & 10 & 7098658 & 7098658,00 & 0,00 & 1 \\
\hline Chr12a & 9552 & 9552 & 10 & 9552 & 9552,00 & 0,00 & 0 \\
\hline Chr12b & 9742 & 9742 & 10 & 9742 & 9742,00 & 0,00 & 0 \\
\hline Chr12c & 11156 & 11156 & 10 & 11156 & 11156,00 & 0,00 & 0 \\
\hline Chr15a & 9896 & 9896 & 10 & 9896 & 9896,00 & 0,00 & 0 \\
\hline Esc16a & 68 & 68 & 10 & 68 & 68,00 & 0,00 & 0 \\
\hline Esc16b & 292 & 292 & 10 & 292 & 292,00 & 0,00 & 0 \\
\hline Esc16c & 160 & 160 & 10 & 160 & 160,00 & 0,00 & 0 \\
\hline Esc16d & 16 & 16 & 10 & 16 & 16,00 & 0,00 & 0 \\
\hline Esc16e & 28 & 28 & 10 & 28 & 28,00 & 0,00 & 0 \\
\hline Esc16f & 0 & 0 & 10 & 0 & 0,00 & 0,00 & 0 \\
\hline Esc16g & 26 & 26 & 10 & 26 & 26,00 & 0,00 & 0 \\
\hline Esc16h & 996 & 996 & 10 & 996 & 996,00 & 0,00 & 0 \\
\hline Esc16i & 14 & 14 & 10 & 14 & 14,00 & 0,00 & 0 \\
\hline Esc16j & 8 & 8 & 10 & 8 & 8,00 & 0,00 & 0 \\
\hline Esc $32 \mathrm{a}$ & 130 & 136 & 01 & 140 & 139,40 & 7,23 & 240 \\
\hline $\operatorname{Esc} 32 b$ & 168 & 168 & 10 & 168 & 168,00 & 0,00 & 0 \\
\hline $\operatorname{Esc} 32 \mathrm{c}$ & 642 & 642 & 10 & 642 & 642,00 & 0,00 & 0 \\
\hline $\operatorname{Esc} 32 d$ & 200 & 200 & 10 & 200 & 200,00 & 0,00 & 0 \\
\hline Esc $32 \mathrm{e}$ & 2 & 2 & 10 & 2 & 2,00 & 0,00 & 0 \\
\hline $\operatorname{Esc} 32 \mathrm{~g}$ & 6 & 6 & 10 & 6 & 6,00 & 0,00 & 0 \\
\hline $\operatorname{Esc} 32 \mathrm{~h}$ & 438 & 438 & 10 & 438 & 438,00 & 0,00 & 0 \\
\hline Esc64a & 116 & 116 & 10 & 116 & 116,00 & 0,00 & 0 \\
\hline Esc128 & 64 & 64 & 10 & 64 & 64,00 & 0,00 & 65 \\
\hline Had12 & 1652 & 1652 & 10 & 1652 & 1652,00 & 0,00 & 0 \\
\hline Had14 & 2724 & 2724 & 10 & 2724 & 2724,00 & 0,00 & 0 \\
\hline Had16 & 3720 & 3720 & 10 & 3720 & 3720,00 & 0,00 & 0 \\
\hline Had18 & 5358 & 5358 & 10 & 5358 & 5358,00 & 0,00 & 1 \\
\hline $\operatorname{Had} 20$ & 6922 & 6922 & 10 & 6922 & 6922,00 & 0,00 & 0 \\
\hline Nug12 & 578 & 578 & 10 & 578 & 578,00 & 0,00 & 0 \\
\hline Nug14 & 1014 & 1014 & 10 & 1014 & 1014,00 & 0,00 & 0 \\
\hline Nug15 & 1150 & 1150 & 10 & 1150 & 1150,00 & 0,00 & 0 \\
\hline Nug16a & 1610 & 1610 & 10 & 1610 & 1610,00 & 0,00 & 0 \\
\hline Nug16b & 1240 & 1240 & 10 & 1240 & 1240,00 & 0,00 & 0 \\
\hline Nug17 & 1732 & 1732 & 10 & 1732 & 1732,00 & 0,00 & 0 \\
\hline Nug18 & 1930 & 1930 & 10 & 1930 & 1930,00 & 0,00 & 0 \\
\hline Nug20 & 2570 & 2570 & 10 & 2570 & 2570,00 & 0,00 & 0 \\
\hline Rou12 & 235528 & 235528 & 10 & 235528 & 235528,00 & 0,00 & 0 \\
\hline Rou15 & 354210 & 354210 & 10 & 354210 & 354210,00 & 0,00 & 0 \\
\hline Rou20 & 725522 & 725522 & 08 & 725582 & 725534,00 & 0,00 & 1 \\
\hline Scr12 & 31410 & 31410 & 10 & 31410 & 31410,00 & 0,00 & 0 \\
\hline Scr15 & 51140 & 51140 & 10 & 51140 & 51140,00 & 0,00 & 0 \\
\hline Scr20 & 110030 & 110030 & 10 & 110030 & 110030,00 & 0,00 & 3 \\
\hline Sko42 & 15812 & 15880 & 01 & 16036 & 15969,00 & 0,99 & 240 \\
\hline Sko49 & 23386 & 23582 & 01 & 23736 & 23652,40 & 1,13 & 240 \\
\hline Tai12a & 224416 & 224416 & 10 & 224416 & 224416,00 & 0,00 & 0 \\
\hline Tai15a & 388214 & 388214 & 10 & 388214 & 388214,00 & 0,00 & 0 \\
\hline Tai15b & 51765268 & 51765268 & 10 & 51765268 & 51765268,00 & 0,00 & 0 \\
\hline Tai17a & 491812 & 491812 & 10 & 491812 & 491812,00 & 0,00 & 0 \\
\hline Tai20a & 703482 & 703482 & 05 & 713260 & 706128,90 & 0,37 & 32 \\
\hline Tai20b & 122455319 & 122455319 & 10 & 122455319 & 122455319,00 & 0,00 & 1 \\
\hline Tai25a & 1167256 & 1181326 & 01 & 1193120 & 1187990,60 & 1,77 & 240 \\
\hline Tai30a & 1818146 & 1841180 & 01 & 1867650 & 1858562,80 & 2,22 & 240 \\
\hline Tail40a & 3139370 & 3215360 & 01 & 3251200 & 3233951,20 & 3,01 & 240 \\
\hline Tail50a & 4938796 & 5084020 & 01 & 5143598 & 5113257,40 & 3,53 & 240 \\
\hline Tho30 & 149936 & 150578 & 01 & 151742 & 151189,20 & 0,83 & 240 \\
\hline Tho40 & 240516 & 243362 & 01 & 246172 & 244773,00 & 1,76 & 240 \\
\hline
\end{tabular}


The program stops when the optimal solution is reached or when the execution time exceeds 240 seconds. We take two digits after the comma, for the results shown in the two columns: Average and the Relative Percentage Deviation \%RPD.

As Table II shows, the proposed algorithm allows to obtain always the optimal solution of $81,25 \%$ of the instances tested in a time not exceeding three seconds. The \%RPD of $93,75 \%$ of the instances does not exceed $2 \%$ and this clearly shows that the GBSA algorithm converges well to the optimal solution. According to the values shown in the Table II, when the value of \%RPD is equal to $0.00 \%$, this means that the program reaches exactly the optimal solution at least 8 times per 10 tests and in this case the best and the worst solution are often the same.

Abd El-Nasser et al. [38] presented a comparative study between Meta-heuristic algorithms: Genetic Algorithm (GA), Tabu Search (TS), and Simulated annealing (SA) for solving a real-life (QAP) and analyze their performance in terms of both runtime efficiency and solution quality [38].

The Fig. 6 compares the relative percentage deviation of some instances of QALIB for our proposed algorithm GBSA, GA, TS and SA. The result shows that GBSA has more quality than the other algorithms for solving the QAP. We can deduce that our proposed method has really improved SA's effectiveness in solving these instances which we have chosen as an example for our comparative study.

There exist two sets of problems in QAPLIB that represent a challenge for any proposed algorithm. These problems were introduced by Skorin-Kapov [39] and Taillard [40].

We selected 9 instances from Skorin-Kapov and 7 instances from Taillard. For this list of QAPLIB instances, we compared our proposed method with others recent methods such as: Memetic algorithm (BMA) [41], Breakout local search (BLS) [42] and Cooperative parallel tabu search algorithm (CPTS) [43]. The list of instances shown in Table III is a challenge for our algorithm.

We have fixed the maximum execution time of GBSA algorithm at 4 minutes. As the results depict (Table III), the GBSA algorithm needs some improvement to better solve some hard instances of QAP. But in
\%RDP of instances for GBSA, GA, TS and SA

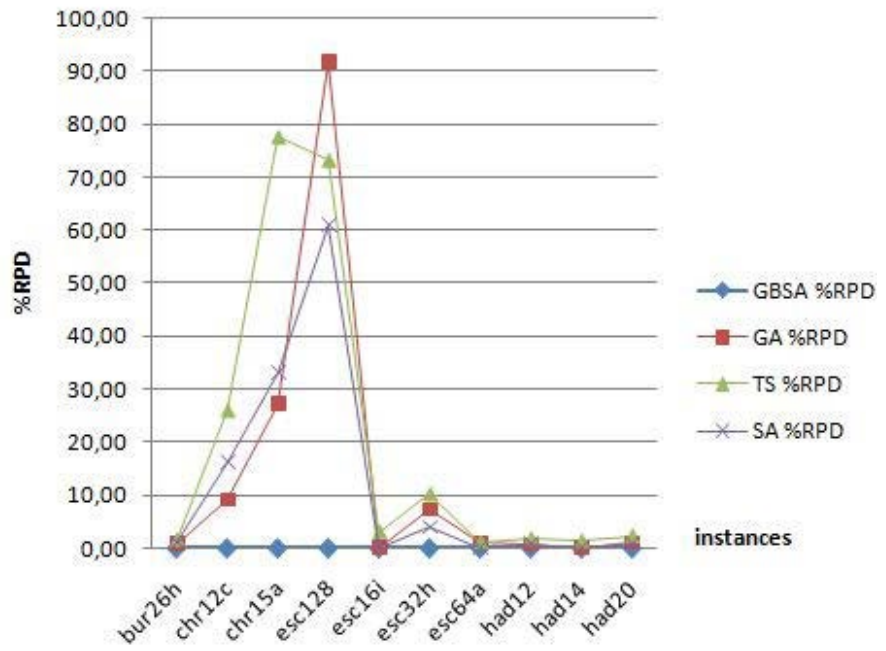

Fig. 6. \%RDP of some instances for GBSA, GA, TS and SA algorithms.

general, the proposed algorithm seems promising to solve the quadratic assignment problem. According to the values of the relative percentage deviation from the best known solution, GBSA algorithm produces results near the global optimum in a reasonable time.

\section{CONCLUSION}

The GBSA algorithm is the result of the hybridization of two methods: golden ball metaheuristic and simulated annealing method. This new hybrid algorithm is based on soccer concepts; it incorporates and guides simulated Annealing technique to escape from the local minima and to find the global optimal solution. This method has never been proposed or tested on QAPLIB instances. In this work we proposed an adaptation of our strategy to solve the QAP. The numerical results indicate the efficiency of the proposed GBSA adaptation and its performance compared to algorithms in literature of QAP. As a result, we deduce that our proposed approach has a high convergence speed.

TABLE III. COMPARISON OF GBSA Algorithm with Algorithms IN THE LiterATURE OF THE QAP

\begin{tabular}{|c|c|c|c|c|c|c|c|c|c|}
\hline \multicolumn{2}{|c|}{} & \multicolumn{2}{|c|}{ GBSA } & \multicolumn{2}{c|}{ BMA } & \multicolumn{3}{c|}{ BLS } & \multicolumn{2}{c|}{ CPTS } \\
\hline Instance & BKS & \%RPD & Time $(\mathrm{m})$ & \%RPD & Time $(\mathrm{m})$ & \%RPD & Time (m) & \%RPD & Time $(\mathrm{m})$ \\
\hline Sko72 & 66256 & 0,543 & 4.0 & 0.000 & 3.5 & 0.000 & 4.1 & 0.000 & 69.6 \\
\hline Sko81 & 90998 & 0,481 & 4.0 & 0.000 & 4.3 & 0.000 & 13.9 & 0.000 & 121.4 \\
\hline Sko90 & 115534 & 0,614 & 4.0 & 0.000 & 15.3 & 0.000 & 16.6 & 0.000 & 193.7 \\
\hline Sko100a & 152002 & 0,539 & 4.0 & 0.000 & 22.3 & 0.001 & 20.8 & 0.000 & 304.8 \\
\hline Sko100b & 153890 & 0,679 & 4.0 & 0.000 & 6.5 & 0.000 & 10.8 & 0.000 & 309.6 \\
\hline Sko100c & 147862 & 0,396 & 4.0 & 0.000 & 12.0 & 0.000 & 15.5 & 0.000 & 316.1 \\
\hline Sko100d & 149576 & 0,760 & 4.0 & 0.006 & 20.9 & 0.001 & 38.9 & 0.000 & 309.8 \\
\hline Sko100e & 149150 & 0,528 & 4.0 & 0.000 & 11.9 & 0.000 & 42.5 & 0.000 & 309.1 \\
\hline Sko100f & 149036 & 0,704 & 4.0 & 0.000 & 23.0 & 0.000 & 17.3 & 0.003 & 310.3 \\
\hline Tai40a & 3139370 & 3,012 & 4.0 & 0.059 & 8.1 & 0.022 & 38.9 & 0.148 & 3.5 \\
\hline Tai50a & 4938796 & 3,532 & 4.0 & 0.131 & 42.0 & 0.157 & 45.1 & 0.440 & 10.3 \\
\hline Tai60a & 7205962 & 2,870 & 4.0 & 0.144 & 67.5 & 0.251 & 47.9 & 0.476 & 26.4 \\
\hline Tai80a & 13499184 & 2,965 & 4.0 & 0.426 & 65.8 & 0.517 & 47.3 & 0.691 & 94.8 \\
\hline Tai100a & 21052466 & 2,771 & 4.0 & 0.405 & 44.1 & 0.430 & 39.0 & 0.589 & 261.2 \\
\hline Tai50b & 458821517 & 0,285 & 4.0 & 0.000 & 1.2 & 0.000 & 2.8 & 0.000 & 13.8 \\
\hline Tai60b & 608215054 & 0,147 & 4.0 & 0.000 & 5.2 & 0.000 & 5.6 & 0.000 & 30.4 \\
\hline
\end{tabular}


Moreover, we need to ameliorate this technique even more for some hard QAPLIB instances. Finally, we plan to apply the GBSA algorithm to TSP and compare it with Random-keys Golden Ball algorithm [44]. We plan also to propose a new hybridization such as mixing Golden Ball algorithm with Tabu Search method.

\section{REFERENCES}

[1] S.Sahni, \& Gonzalez, T. P-complete approximation problems. Journal of the ACM (JACM), 23(3), 555-565, (1976).

[2] T.C.Koopmans, \& M. Beckmann, Assignment problems and the location of economic activities. Econometrica: journal of the Econometric Society, 53-76, (1957).

[3] AN.Elshafei, Hospital layout as a quadratic assignment problem. Operational Research Quarterly, 167-179, (1977).

[4] A. M.Geoffrion, \& G. W. Graves, Scheduling parallel production lines with changeover costs: Practical application of a quadratic assignment/LP approach. Operations Research, 24(4), 595-610, (1976).

[5] I. Ugi, J. Bauer, J. Brandt, J. Friedrich, J. Gasteiger, C. Jochum, \& W. Schubert, Neue anwendungsgebiete für computer in der chemie. Angewandte Chemie, 91(2), 99-111, (1979).

[6] Z. Drezner, Extensive experiments with hybrid genetic algorithms for the solution of the quadratic assignment problem. Computers \& Operations Research, 35(3), 717-736, (2008).

[7] Y. Hani, L. Amodeo, F. Yalaoui, \& H. Chen, Ant colony optimization for solving an industrial layout problem. European Journal of Operational Research, 183(2), 633-642, (2007).

[8] L. Y. Tseng, \& S. C. Liang, A hybrid metaheuristic for the quadratic assignment problem. Computational Optimization and Applications, 34(1), 85-113, (2006).

[9] V.Černý, Thermodynamical approach to the traveling salesman problem: An efficient simulation algorithm. Journal of Optimization Theory and Applications, 45(1), 41-51, (1985).

[10] R.E.Burkard, E. Cela, P.M.Pardalos, \& L.S.Pitsoulis. The quadratic assignment problem. In Handbook of Combinatorial Optimization (pp. 1713-1809). Springer US, (1998).

[11] V. Kaibel, Polyhedral combinatorics of QAPs with less objects than locations. Univ., (1997).

[12] Z. Drezner, Compounded genetic algorithms for the quadratic assignment problem. Operations Research Letters, 33(5), 475-480, (2005).

[13] M. A. El-Baz, A genetic algorithm for facility layout problems of different manufacturing environments. Computers \& Industrial Engineering, 47(2), 233-246, (2004).

[14] W. A. N. G. Rong-Long, \& K. Okazaki, Solving facility layout problem using an improved genetic algorithm. IEICE Transactions on Fundamentals of Electronics, Communications and Computer Sciences, 88(2), 606-610, (2005).

[15] Z. Drezner, The extended concentric tabu for the quadratic assignment problem. European Journal of Operational Research, 160(2), 416-422, (2005).

[16] T. James, C. Rego, \& F. Glover, A cooperative parallel tabu search algorithm for the quadratic assignment problem. European Journal of Operational Research, 195(3), 810-826, (2009).

[17] A. Misevicius, A tabu search algorithm for the quadratic assignment problem. Computational Optimization and Applications, 30(1), 95-111, (2005).

[18] H. Q. Saremi, B. Abedin, \& A. M. Kermani, Website structure improvement: Quadratic assignment problem approach and ant colony meta-heuristic technique. Applied Mathematics and Computation, 195(1), 285-298, (2008).

[19] H. Liu, A. Abraham, \& J. Zhang, A particle swarm approach to quadratic assignment problems. In Soft Computing in Industrial Applications (pp. 213-222). Springer Berlin Heidelberg, (2007).

[20] M. Bashiri, \& H. Karimi. Effective heuristics and meta-heuristics for the quadratic assignment problem with tuned parameters and analytical comparisons. Journal of Industrial Engineering International, 8(1), 1-9, (2012).

[21] G. Paul.Comparative performance of tabu search and simulated annealing heuristics for the quadratic assignment problem. Operations Research
Letters, 38(6), 577-581, (2010).

[22] E. Osaba, F. Diaz, \& E. Onieva, A novel meta-heuristic based on soccer concepts to solve routing problems. In Proceedings of the 15th Annual Conference Companion on Genetic and Evolutionary Computation (pp. 1743-1744). ACM, (2013, July).

[23] E. Osaba, F. Diaz, R. Carballedo, E. Onieva, \& A. Perallos, Focusing on the Golden Ball Metaheuristic: An Extended Study on a Wider Set of Problems. The Scientific World Journal, 2014, (2014).

[24] A. M. Frieze, G. Galbiati , and F. Maffioli, On the worst-case performance of some algorithms for the asymmetric traveling salesman problem, Networks, 12 (1), 23-39, (1982).

[25] B. Golden, E. Baker, J. Alfaro, and J. Schaffer, The vehicle routing problem with backhauling: two approaches, in Proceedings of the 21st Annual Meeting of SE TIMS, pp. 90-92, South Carolina, SC, USA, (1985).

[26] E.Osaba, \& F. Díaz. Design and Implementation of a Combinatorial Optimization Multi-population Meta-heuristic for Solving Vehicle Routing Problems. International Journal of Interactive Multimedia and Artificial Intelligence, 4(2), 89-90, (2016).

[27] I. Rivin, I. Vardi, and P. Zimmermann, The n-queens problem, The American Mathematical Monthly, 101(7), pp. 629-639, (1994).

[28] S. Martello, and P. Toth, Knapsack Problems: Algorithms and Computer Implementations, Wiley, New York, NY, USA, (1990).

[29] F. Sayoti, \& M. E. Riffi, Golden Ball Algorithm for solving Flow Shop Scheduling Problem. International Journal of Artificial Intelligence and Interactive Multimedia, 4(1), 15-18, (2016).

[30] F. Sayoti, M. E. Riffi, \& H. LabaniOptimization of Makespan in Job Shop Scheduling Problem by Golden Ball Algorithm. Indonesian Journal of Electrical Engineering and Computer Science, 4(3), (2016).

[31] A. Misevičius, A modified simulated annealing algorithm for the quadratic assignment problem. Informatica, 14(4), 497-514, (2003).

[32] G. A. Croes, A method for solving traveling-salesman problems. Operations Research, 6(6), 791-812, (1958).

[33] S. Lin, Computer solutions of the traveling salesman problem. Bell System Technical Journal, 44(10), 2245-2269, (1965).

[34] M. Fischetti, Salazar Gonzalez, J. J., \& Toth, P.. A branch-and-cut algorithm for the symmetric generalized traveling salesman problem. Operations Research, 45(3), 378-394, (1997).

[35] C. D. Tarantilis, Solving the vehicle routing problem with adaptive memory programming methodology. Computers \& Operations Research, 32(9), 2309-2327, (2005).

[36] R.E.Burkard,S. Karisch, \& F.Rendl. QAPLIB-A quadratic assignment problem library. European Journal of Operational Research, 55(1), 115119, (1991).

[37] W. Ellen E., C.Roger D., \& F.Mark A. Parallel simulated annealing using speculative computation. IEEE Transactions on Parallel and Distributed Systems, 1991, 2(4), 483-494.

[38] G.A, El-Nasser A., A.M. Mahmoud, \& E.-S. M. El-Horbaty. A comparative study of meta-heuristic algorithms for solving quadratic assignment problem. International Journal of Advanced Computer Science and Applications, 5(1), arXiv:1407.4863, (2014).

[39] J.Skorin-Kapov. Tabu search applied to the quadratic assignment problem. ORSA Journal on computing, 2(1), 33-45,(1990).

[40] E.Taillard. Robust taboo search for the quadratic assignment problem. Parallel computing, 17(4-5), 443-455, (1991).

[41] U.Benlic, \& J.K.Hao. Memetic search for the quadratic assignment problem. Expert Systems with Applications, 42(1), 584-595, (2015).

[42] U. Benlic, \& J.K. Hao. Breakout local search for the quadratic assignment problem. Applied Mathematics and Computation, 219(9), 4800-4815, (2013).

[43] T.James, C. Rego., \& F.Glover. A cooperative parallel tabu search algorithm for the quadratic assignment problem. European Journal of Operational Research, 195(3), 810-826, (2009).

[44] F. Sayoti, \& M. E. Riffi, Random-Keys Golden Ball Algorithm for Solving Traveling Salesman Problem. International Review on Modelling and Simulations (IREMOS), 8(1), 84-89, (2015). 


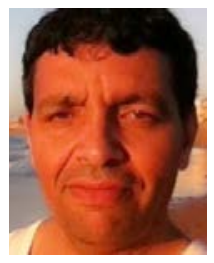

Mohammed Essaid Riffi

Ms. Mohammed Essaid Riffi is a professor at the University of Chouaib Doukkali, El Jadida, Morocco, currently he is chief of Computer Science department and former coordinator of Mathematical and Computer Science. Its research axis is the metaheuristics.

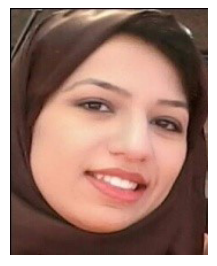

Fatima Sayoti

Ms. Fatima Sayoti (15-02-1988) received a master's degree of software quality from University of Chouaib Doukkali, El Jadida, Morocco in 2011. She is currently pursuing his Ph.D. degree (Computer Science) at the University of Chouaib Doukkali, Faculty of Sciences, El Jadida, Morocco. She is also a temporary teacher at Polydisciplinary Faculty, El Jadida (Morocco). 